Muriel Augustini

Mr Pascal Duret

Paul Irlinger

Catherine Louveau

\title{
Pratiques sportives des enfants et rôle socialisateur du sport
}

In: Enfance. Tome 47 n²-3, 1994. pp. 171-185.

Citer ce document / Cite this document :

Augustini Muriel, Duret Pascal, Irlinger Paul, Louveau Catherine. Pratiques sportives des enfants et rôle socialisateur du sport. In: Enfance. Tome 47 n²-3, 1994. pp. 171-185.

http://www.persee.fr/web/revues/home/prescript/article/enfan_0013-7545_1994_num_47_2_2097 


\section{Abstract}

This article is based upon the data collected after a survey organized by the agf (Assurances générales de France) in 1991. A sample of 4465 young people aged 8 to 18 volunteered to answer questionnaires which were collected thereafter.

Studying the responses led to framing the young people's sport practises. The young people's sexual and social patterns, just like the adult's, strongly determine whether they will or won't practise sport and remarkably underline the selected type of sport activity as well as the conditions of practice. These results also make it clear that sport plays a great part in children's socialization through their sporting ideology and the way they identify with champions. The young generally hold to the great principles of tradi- tionnal sport ethics, but they also take into account the social reality of sport conveyed by the mass media. The fact that chidren refer to such a big number of champions leads to diversified figures of success. The media play a great part in bringing champions closer to children but they also make them more ephemeral.

\section{Résumé}

Cet article s'appuie sur les données d'une enquête diffusée en 1991 à l'occasion d'un concours national organisé par les agf (Assurances générales de France). 4465 questionnaires ont été recueillis et correspondent aux réponses d'un échantillon spontané déjeunes âgés de 8 à 18 ans.

L'exploitation de cette enquête a permis de tracer le panorama des pratiques sportives des jeunes. A l'instar des adultes, les modèles de sexe et les modèles sociaux infèrent sur les probabilités d'accès à la pratique et marquent notablement les choix et les modalités de cette pratique. Les résultats permettent également d'éclairer le rôle du sport dans le processus de socialisation des enfants à travers leur idéologie sportive et leur identification aux champions. Globalement, les jeunes adhèrent largement aux grands principes de l'éthique sportive traditionnelle, mais ils prennent également en compte la réalité sociale du sport véhiculée par les mass media. La profusion des champions évoqués par les enfants conduit à une diversification des figures de la réussite. Les médias concourent à rendre les champions plus proches et plus éphémères. 


\title{
Pratiques sportives des enfants et rôle socialisateur du sport
}

\author{
M. Augustini \\ P. Duret \\ P. Irlinger \\ C. Louveau*
}

\section{RESUME}

Cet article s'appuie sur les donnèes d'une enquête diffusée en 1991 à l'occasion d'un concours national organisé par les AGF (Assurances générales de France). 4465 questionnaires ont été recueillis et correspondent aux réponses d'un échantillon spontané de jeunes âgés de 8 à 18 ans.

L'exploitation de cette enquête a permis de tracer le panorama des pratiques sportives des jeunes. A l'instar des adultes, les modèles de sexe et les modèles sociaux infêrent sur les probabilités d'accès à la pratique et marquent notablement les choix et les modalités de cette pratique. Les résultats permettent également d'éclairer le rôle du sport dans le processus de socialisation des enfants à travers leur idéologie sportive et leur identification aux champions. Globalement, les jeunes adhèrent largement aux grands principes de l'éthique sportive traditionnelle, mais ils prennent également en compte la réalité sociale du sport véhiculée par les mass media. La profusion des champions évoqués par les enfants conduit à une diversification des figures de la réussite. Les médias concourent à rendre les champions plus proches et plus éphémères.

Mots clés : Sociologie. Sport. Socialisation. Enfants.

\section{SUM M A R Y}

This article is based upon the data collected after a survey organized by the AGF (Assurances générales de France) in 1991. A sample of 4465 young people aged 8 to 18 volunteered to answer questionnaires which were collected thereafter.

Studying the responses led to framing the young people's sport practises. The young people's sexual and social patterns, just like the adult's, strongly determine whether they will or won't practise sport and remarkably underline the selected type of sport activity as well as the conditions of practice. These results also make it clear that sport plays a great part in children's socialization through their sporting ideology and the way they identify with champions. The young generally hold to the great principles of traditionnal sport ethics, but they also take into account the social reality of sport conveyed by the mass media. The fact that chidren refer to such a big number of champions leads to diversified figures of success. The media play a great part in bringing champions closer to children but they also make them more ephemeral.

* Laboratoire de Sociologie, Mission Recherche de l'Institut national du sport et de l'éducation physique, 11 av. du Tremblay, 75012 Paris.

ENFANCE, ${ }^{\circ} 2-3 / 1994$, p. 171 à 185 


\section{INTRODUCTION}

Le rôle du sport dans la socialisation des jeunes, souvent considéré comme une évidence, est pourtant une question complexe. En effet, la socialisation au moyen du sport a longtemps été assimilée, dans une perspective pédagogique, soit au simple respect des règles du « jeu " sportif (règlements, arbitre, entraîneur...), soit aux bienfaits d'une pratique amenant à s'insérer dans des collectifs, à apprendre à perdre ou à gagner dans un esprit de "fair-play ". Mais elle prend également appui sur les champions pour suggérer, à travers ces modèles, des lignes d'identification. En outre, les négociations au sein des équipes et la multiplication des expériences des jeunes entre eux, sans référence au monde adulte, peuvent tout aussi bien être invoquées (Galland, 1991). Enfin, on peut débusquer la socialisation dans le travail invisible d'intériorisation de normes implicites (Vigarello, 1977). Cette transmission des valeurs et des attitudes passe alors davantage par le processus informel d'un "curriculum caché " (Forquin, 1993) que par des modalités rationnelles et délibérées.

Pour éclairer sans a priori théorique les fonctions du sport dans le processus de socialisation, il faut disposer d'une vision précise de ce que représente aujourd'hui sa pratique pour les jeunes. Dans cette perspective, nous avons exploité une enquête par questionnaire conçue par l'Institut de l'enfance et de la famille et diffusée par l'agence " Ricochets " dans le cadre d'une opération de communication en direction des jeunes par les AGF, partenaire olympique des Jeux d'Albertville (Duret, Irlinger, Louveau, Vulbeau, 1993). Entre décembre 1990 et janvier 1991, 4465 questionnaires ont été recueillis, correspondant aux réponses d'un échantillon "spontané " de jeunes âgés de 8 à 18 ans. La population des jeunes qui ont répondu à l'enquête présente les caractéristiques suivantes :

- le nombre de garçons et de filles est à peu près équivalent (ils forment respectivement 48 et $52 \%$ de l'échantillon);

- $53 \%$ sont des élèves d'école primaire, $39 \%$ sont des collégiens et $7 \%$ des lycéens;

- les provinciaux des petites villes sont surreprésentés (28\%), les Parisiens sous-représentés $(12 \%)$;

- au niveau de l'origine sociale, les enfants de cadres et professions intellectuelles supérieures sont surreprésentés $(17 \%)$ et ceux des professions intermédiaires sous-représentés $(9 \%)$; la distribution des enfants des autres catégories sociales est proche de la distribution nationale.

Cette population relativement jeune et bien insérée dans le cadre scolaire appartient à la jeunesse traditionnelle.

Dans un premier temps, cette étude permet de tracer le panorama des modalités et des finalités de la pratique sportive de ces jeunes. Elle décrit 
aussi comment les enfants se positionnent par rapport aux normes traditionnelles de l'éthique sportive. Enfin, elle amène à s'interroger sur le pouvoir de socialisation que véhiculent les champions, mis au premier plan par les adultes et réappropriés par les enfants.

\section{I - PANORAMA DES PRATIQUeS SPORTIVES DES ENFANTS}

\section{Données générales}

Tous les jeunes ou presque s'adonnent au sport : $83 \%$ d'entre eux déclarent en faire au moins une fois par semaine en dehors des cours d'Education physique et sportive, $62 \%$ pratiquent une à trois heures par semaine, $21 \%$ de quatre à six heures. Dans la majorité des cas, cette pratique se déroule dans un environnement encadré et institutionnalisé : $70 \%$ des pratiquants exercent au moins une de leurs activités sportives dans un club.

Les enfants vivent généralement leur engagement sportif comme un libre choix ou comme le résultat d'une négociation; seulement $6 \%$ d'entre eux estiment que cette activité leur a été imposée. $87 \%$ des pratiquants sont satisfaits de leur discipline sportive, les enfants qui ont participé à la décision ayant un indice de satisfaction plus élevé que les autres.

Concernant les finalités de la pratique sportive, les jeunes estiment que si l'on fait du sport c'est :

— pour être en bonne santé : $50 \%$;

— pour être fort, célèbre, le premier ou gagner de l'argent : $9 \%$;

- pour rencontrer d'autres personnes : $9 \%$;

- pour avoir un beau corps : $6 \%$.

Le tableau suivant visualise les sports les plus pratiqués par les jeunes de l'enquête.

TABLEAU 1. - Sports pratiqués le plus fréquemment par les jeunes de l'enquête

\begin{tabular}{|c|l|c|c|l|c|}
\hline Rang & $\begin{array}{c}\text { Disciplines } \\
\text { sportives }\end{array}$ & $\begin{array}{c}\text { Effectif } \\
(\%)\end{array}$ & Rang & $\begin{array}{c}\text { Disciplines } \\
\text { sportives }\end{array}$ & $\begin{array}{c}\text { Effectif } \\
(\%)\end{array}$ \\
\hline $1^{\circ}$ & Football & 19,8 & $11^{\circ}$ & Basket ball & 6,8 \\
\hline $2^{\circ}$ & Vélo & 16,8 & $12^{\circ}$ & Hand ball & 4,8 \\
\hline $3^{\circ}$ & Tennis & 16,5 & $13^{\circ}$ & Volley ball & 4,1 \\
\hline $4^{\circ}$ & Natation & 15,2 & $14^{\circ}$ & Equitation & 3,9 \\
\hline $5^{\circ}$ & Danse & 10,5 & $15^{\circ}$ & Rugby & 2,7 \\
\hline $6^{\circ}$ & Gymnastique & 9,5 & $16^{\circ}$ & Ping-pong & 2,4 \\
\hline $7^{\circ}$ & Marche & 8,2 & $17^{\circ}$ & Patinage & 2,1 \\
\hline $8^{\circ}$ & Ski & 7,4 & $18^{\circ}$ & Karaté & 1,5 \\
\hline $9^{\circ}$ & Course & 7,2 & $19^{\circ}$ & Athlétisme & 1,3 \\
\hline $10^{\circ}$ & Judo & 7 & $20^{\circ}$ & Rollers & 0,7 \\
\hline
\end{tabular}


Ces données générales sont largement modulées en fonction des caractéristiques des enfants : le sexe, l'âge, qui peut être apprécié par le niveau scolaire, et la position sociale, appréhendée par la catégorie socioprofessionnelle des parents, influencent notablement la pratique sportive des jeunes.

\section{Variations de la pratique selon le sexe}

Les garçons sont plus engagés dans le sport que les filles : $86 \%$ d'entre eux s'adonnent à une activité physique contre $80 \%$ des filles ; $30 \%$ des pratiquants y consacrent plus de quatre heures par semaine contre $19 \%$ des pratiquantes. L'institutionnalisation de la pratique diffère également selon le sexe : $77 \%$ des garçons contre $64 \%$ des filles pratiquent leur(s) sport(s) dans le cadre d'un club.

Concernant les finalités de la pratique, garçons et filles s'accordent majoritairement sur le fait "d'être en bonne santé " $(50 \%)$; au-delà de ce consensus, les uns et les autres affirment leur spécificité : $15 \%$ des garçons pensent que si l'on fait du sport c'est « pour être le plus fort, le premier, être célèbre ou gagner de l'argent ", quand c'est le cas de $4 \%$ des filles; celles-ci font davantage du sport " pour avoir un beau corps " $(9 \%$ des filles le pensent pour à peine $4 \%$ des garçons).

Enfin, les disciplines dans lesquelles s'engagent préférentiellement les enfants diffèrent selon le sexe. Le football est aux garçons ce que la danse est aux filles : leur pratique d'élection. De plus, lorsque la pratique sportive choisie est commune aux deux sexes, garçons et filles ne s'y engagent pas dans les mêmes proportions comme le montre le tableau suivant.

Ainsi, garçons et filles conçoivent différemment l'activité physique. Les différences se situent tant au niveau de la discipline choisie que dans les modalités et les finalités de sa pratique.

TABleAu 2. - Sports pratiqués le plus fréquemment par les filles et les garçons

\begin{tabular}{|c|l|c|c|l|c|}
\hline \multicolumn{3}{|c|}{ Garçons } & \multicolumn{3}{c|}{ Filles } \\
\hline Rang & $\begin{array}{c}\text { Disciplines } \\
\text { sportives }\end{array}$ & $\begin{array}{c}\text { Effectif } \\
(\%)\end{array}$ & Rang & $\begin{array}{c}\text { Disciplines } \\
\text { sportives }\end{array}$ & $\begin{array}{c}\text { Effectif } \\
(\%)\end{array}$ \\
\hline $1^{\circ}$ & Football & 45 & $1^{\circ}$ & Danse & 24 \\
\hline $2^{\circ}$ & Tennis & 21 & $2^{\circ}$ & Natation & 21 \\
\hline $3^{\circ}$ & Vélo & 19 & $3^{\circ}$ & Vélo & 20 \\
\hline $4^{\circ}$ & Natation & 15 & $4^{\circ}$ & Gymnastique & 19 \\
\hline $5^{\circ}$ & Judo & 13 & $5^{\circ}$ & Tennis & 18 \\
\hline $6^{\circ}$ & Courses & 10 & $6^{\circ}$ & Marche & 13 \\
\hline $7^{\circ}$ & Skj & 9 & $7^{\circ}$ & Basket-ball & 9 \\
\hline $8^{\circ}$ & Basket-ball & 7 & $8^{\circ}$ & Ski & 9 \\
\hline $9^{\circ}$ & Marche & 6 & $9^{\circ}$ & Hand-ball & 7 \\
\hline $10^{\circ}$ & Ping-pong & 4 & $10^{\circ}$ & Volley-ball & 7 \\
\hline
\end{tabular}




\section{Variations de la pratique selon le niveau scolaire}

L'engagement dans la pratique sportive croît avec l'âge, apprécié par le niveau scolaire des enfants : $87 \%$ des lycéens font du sport contre $83 \%$ des collégiens et $82 \%$ des élèves du primaire. Les lycéens y consacrent aussi davantage de temps que les plus jeunes : $39 \%$ d'entre eux pratiquent un sport quatre à six heures par semaine quand c'est le cas de $32 \%$ des collégiens et de $18 \%$ des élèves du primaire.

Plus l'âge augmente, plus l'institutionnalisation de la pratique diminue : $72 \%$ des enfants du primaire et $71 \%$ des collégiens pratiquants s'adonnent à leur sport dans un club pour seulement $59 \%$ des lycéens.

Quel que soit le niveau scolaire, la finalité première accordée au sport reste " être en bonne santé ". "Etre fort, le premier, devenir célèbre ou gagner de l'argent " intéresse surtout les plus jeunes ( $12 \%$ des élèves du primaire, $3 \%$ des lycéens), les plus âgés voyant davantage dans le sport le moyen de « rencontrer d'autres personnes » (14\% des lycéens pour $6 \%$ d'élèves du primaire).

Si les pratiques favorites des enfants restent quasiment identiques selon le niveau scolaire, ils ne s'y engagent pas dans les mêmes proportions. Le tableau suivant montre en particulier que le football, pratique de prédilection des plus jeunes, cède peu à peu la place au tennis.

TABleau 3. - Sports pratiqués le plus fréquemment selon le niveau scolaire

\begin{tabular}{|c|l|c|l|c|l|c|}
\hline \multirow{3}{*}{ Rang } & \multicolumn{2}{|c|}{ Primaire } & \multicolumn{2}{c|}{ Collège } & \multicolumn{2}{c|}{ Lyc6e } \\
\cline { 2 - 7 } & $\begin{array}{c}\text { Disciplines } \\
\text { sportives }\end{array}$ & $\begin{array}{c}\text { Effectif } \\
(\%)\end{array}$ & $\begin{array}{c}\text { Disciplines } \\
\text { sportives }\end{array}$ & $\begin{array}{c}\text { Effectif } \\
(\%)\end{array}$ & $\begin{array}{c}\text { Disciplines } \\
\text { sportives }\end{array}$ & $\begin{array}{c}\text { Effectif } \\
(\%)\end{array}$ \\
\hline $1^{\circ}$ & Football & 22,7 & Tennis & 19,7 & Tennis & 21,8 \\
\hline $2^{\circ}$ & Vélo & 17 & Football & 17 & Vélo & 16,8 \\
\hline $3^{\circ}$ & Natation & 14,7 & Vélo & 16,3 & Natation & 14,9 \\
\hline $4^{\circ}$ & Tennis & 13,2 & Natation & 15,5 & Course & 12,1 \\
\hline $5^{\circ}$ & Danse & 10,8 & Danse & 10,8 & Football & 11,8 \\
\hline
\end{tabular}

\section{Variations de la pratique selon le milieu social}

L'origine sociale des jeunes pèse tout d'abord sur la probabilité d'accéder à la pratique d'un sport. Les enfants de cadres et professions intellectuelles supérieures et ceux des membres des professions intermédiaires ont le taux de pratique le plus élevé ( $89 \%$ ), les enfants d'ouvriers, le moins élevé $(78 \%)$. Notons ici que les enfants qui ont une mère au foyer connaissent, comparés à ceux ayant une mère active et quel que soit son métier, un moindre taux de pratique : $79 \%$ d'entre eux font du sport. La pratique des filles y est plus sensible que celle des garçons : avec les filles d'ouvriers, les filles des mères au foyer ont le taux de pratique le moins élevé $(75 \%)$, ce sont aussi elles qui sont le moins fréquemment inscrites en club, et qui ont le 
moins participé à la décision de faire du sport, elles sont du même coup, un peu moins satisfaites que les autres de cette pratique. $A$ contrario, l'activité professionnelle de la mère se traduit positivement sur l'activité physique. Ces résultats concernant la pratique sportive permettent de formuler l'hypothèse selon laquelle l'activité de la mère hors du foyer induit une plus grande probabilité d'activité pour leurs filles (De Singly, 1987).

C'est pour les enfants de cadres et professions intellectuelles supérieures que la pratique est le plus fréquemment instituée ( $77 \%$ des pratiquants sont en club), a fortiori quand la mère appartient à cette ca tégorie sociale $(79 \%)$; c'est pour les enfants d'ouvriers qu'elle l'est le moins (66\% d'entre eux pratiquent leur sport en club), surtout quand la mère est elle-même ouvrière $(62 \%)$. Ici, ce n'est pas tant l'engagement financier qui importe mais plutôt le style éducatif des parents. Les familles bien dotées en capital scolaire et culturel privilégient la planification des loisirs de leurs enfants alors que les groupes sociaux moins favorisés encouragent l'auto-organisation à proximité du domicile. D'un côté on « fait faire ", on programme, de l'autre on « laisse faire ".

Quelle que soit l'origine sociale, la santé est la raison première citée par les jeunes pour faire du sport, mais les enfants d'agriculteurs et d'ouvriers l'invoquent le plus fréquemment. Etre fort, le premier, gagner de l'argent ou être célèbre grâce au sport est surtout choisi par les enfants d'ouvriers, d'artisans, commerçants et chefs d'entreprise, alors que les enfants de cadres ou de professions intermédiaires ( $\mathrm{y}$ compris les garçons) sont ceux qui citent le moins cette finalité.

L'engagement des jeunes dans les disciplines sportives diffère selon leur milieu social comme le montre le tableau suivant.

Tableau 4. -- Sports pratiqués selon la csP du père (\%)

\begin{tabular}{|l|c|c|c|c|c|c|c|c|c|}
\hline \multicolumn{1}{|c|}{ DIScIPUNES } & Agricult. & $\begin{array}{c}\text { Artisans. } \\
\text { chefs } \\
\text { d'entr. }\end{array}$ & $\begin{array}{c}\text { Prof. intell. } \\
\text { et cadres } \\
\text { supérieurs }\end{array}$ & $\begin{array}{c}\text { Prof. } \\
\text { interméd. }\end{array}$ & Employés & Ouvriers & Retraités & Inactifs & TOTAL \\
\hline Football & 17 & 17 & 12 & 11 & 15 & 20 & 15 & 15 & 17 \\
\hline Vélo & 21 & 18 & 15 & 13 & 15 & 19 & 36 & 16 & 16,8 \\
\hline Tennis & 21 & 14 & 25 & 19 & 18 & 11 & 23 & 7 & 16.5 \\
\hline Natation & 11 & 11 & 17 & 18 & 18 & 14 & 22 & 7 & 15 \\
\hline Danse & 7 & 12 & 13 & 11 & 12 & 8 & 10 & 12 & 10,5 \\
\hline Gymnastique & 13 & 8 & 10 & 11 & 10 & 10 & 10 & 9 & 9,8 \\
\hline Marche & 5 & 7 & 7 & 8 & 8 & 9 & 7 & 12 & 8,2 \\
\hline Skj & 7 & 7 & 10 & 9 & 7 & 5 & 5 & 4 & 7,4 \\
\hline Courses à pied & 11 & 4 & 6 & 7 & 7 & 8 & 15 & 7 & 7 \\
\hline Judo & 3 & 6 & 7 & 7 & 9 & 6 & 2 & 7 & 7 \\
\hline Basket & 9 & 7 & 9 & 8 & 5 & 6 & 7 & 12 & 6,8 \\
\hline Handball & 7 & 4 & 6 & 5 & 4 & 5 & 0 & 4 & 4,8 \\
\hline Volley & 3 & 4 & 4 & 7 & 4 & 4 & 5 & 6 & 4 \\
\hline Equitation & 4 & 4 & 7 & 4 & 4 & 2 & 2 & 1 & 3,9 \\
\hline Rugby & 2 & 3 & 3 & 1 & 3 & 2 & 0 & 7 & 2,7 \\
\hline Ping-pong & 3 & 1 & 3 & 3 & 3 & 3 & 5 & 1 & 2,3 \\
\hline Effectifs totaux & 183 & 434 & 672 & 361 & 954 & 1270 & 39 & 68 & $4465 \cdot$ \\
\hline$\%$ de la pop. d'ensemble & $4 \%$ & $10 \%$ & $15 \%$ & $8 \%$ & $21 \%$ & $28 \%$ & $1 \%$ & $2 \%$ & $89 \% \cdot$ \\
\hline
\end{tabular}

- manquent les enfants dont le père est décédé (32) ou qui n'ont pas répondu à la CSP du père (452).

N.B. : Le total par colonne excède $100 \%$ car les enfants font parfois plusieurs sports. 


\section{Prégnance de modèles et reproduction sociale}

En rapprochant ces résultats de ceux de l'enquête sur "Les pratiques sportives des Français ${ }^{1}$, on constate des analogies évidentes entre les pratiques sportives des jeunes et celles des adultes. Les modèles de sexe et les modèles sociaux qui marquent les pratiques sportives des adultes agissent de la même manière sur celles des enfants.

C'est à l'instar de leurs pères et mères que garçons et filles appréhendent différemment l'univers sportif. L'engagement dans le sport est plus important, plus intensif et plus institutionnalisé pour les hommes ${ }^{2}$ comme pour les garçons. Le sport représente un potentiel d'action et de réussite pour ces derniers, un potentiel de séduction pour les filles ; on retrouve ici des caractéristiques relativement constantes liées au masculin d'une part, au féminin d'autre part (Davisse et Louveau, 1991).

La prégnance des modèles sociaux est également visible dans ces résultats. Pour les jeunes comme pour leurs aînés, les milieux sociaux favorisés ont une probabilité d'accès à la pratique beaucoup plus importante ${ }^{3}$. Pour chaque catégorie socioprofessionnelle, il existe de fortes similitudes entre les choix opérés par les adultes et ceux réalisés par les jeunes concernant leur discipline sportive : les enfants d'ouvriers optent préférentiellement pour le football et le vélo... comme le font les ouvriers eux-mêmes; de même, les enfants de cadres pratiquent surtout du tennis... comme les hommes et femmes cadres.

L'activité physique des jeunes s'inscrit dans une évolution sociale générale. A l'instar de leurs aînés, il est impossible aujourd'hui de réduire l'activité sportive des jeunes à une pratique en club, encadrée. Le développement des pratiques « informelles», entendons celles qui s'effectuent hors de structures sportives traditionnelles, est l'une des caractéristiques majeures de l'évolution récente de la pratique physique : $54 \%$ des Français «bougent " sans détenir de licence. En ce qui concerne les enfants, un quart de ceux qui pratiquent un sport le font de manière informelle, mais cette proportion augmente au fur et à mesure que le jeune s'autonomise pour atteindre $59 \%$ pour les lycéens. Quel que soit l'âge des acteurs, le sport ne saurait échapper aux changements sociaux et culturels ayant, cette dernière décennie, affecté tant les institutions que les

1. P. Irlinger, C. Louveau, M. Metoudi, Les pratiques sportives des Français ; enquête sur les usages sportifs du temps libéré, Paris, INSEP, 1987, 2 t.

2. L'enquête sur les pratiques sportives des Français a montré que le taux de pratique sportive est de $77 \%$ pour les hommes et de $71 \%$ pour les femmes. La differenciation sexuelle existe surtout dans l'institutionnalisation des pratiques sportives des adultes : $34 \%$ des pratiquants et seulement $18 \%$ des pratiquantes détiennent une licence.

3. Pour l'ensemble de la population française, les taux de pratique les plus élevés reviennent aux cadres et professions intellectuelles supérieures (91\%) et aux membres des professions intermédiaires $(85 \%)$, tandis que les agriculteurs et les ouvriers ont les taux de pratique les plus faibles (égalant respectivement $53 \%$ et $67 \%$ ). 
mentalités ; la personnalisation, l'individualisation des pratiques sont le corollaire de leur désinstitutionnalisation (Lipovetsky, 1983).

Des enfants aux adultes, chaque population se donne à voir un peu comme le miroir de l'autre. Y transparaît ce qui se transmet, change ou perdure, au point que l'on peut parler d'une reproduction sociale des pratiques. Celle-ci n'est pas le résultat d'une action délibérée de la part des adultes; elle procède plutôt de modes de rapports au corps - dispositions sexuellement et socialement différenciées précocement intériorisée. Analysant les loisirs des jeunes en général, M. Bozon (1990) observe « une différenciation précoce des activités et des goûts "; " le temps libre des jeunes, note-t-il, joue un rôle important et spécifique dans la reproduction et la consolidation des différences entre sexes et entre groupes sociaux $»$. Le sport ne se distingue pas des autres activités de loisir de ce point de vue.

II - LES ENFANTS ET LIDEOLOGIE SPORTIVE

Le processus de socialisation concerne d'une manière centrale la construction de la capacité de jugement, la prise de position personnelle par rapport à des normes extérieures étant essentielle. Il paraissait alors intéressant de déterminer dans quelle mesure les enfants s'approprient les grands principes de l'éthique sportive. Pour ce faire, nous les avons incités à porter des jugements sur des pratiques que l'éthique sportive réprouve : le dopage, la violence des sportifs et des supporters, les gains des sportifs'. La figure suivante permet de visualiser les résultats concernant l'attitude des jeunes face à ces thèmes.

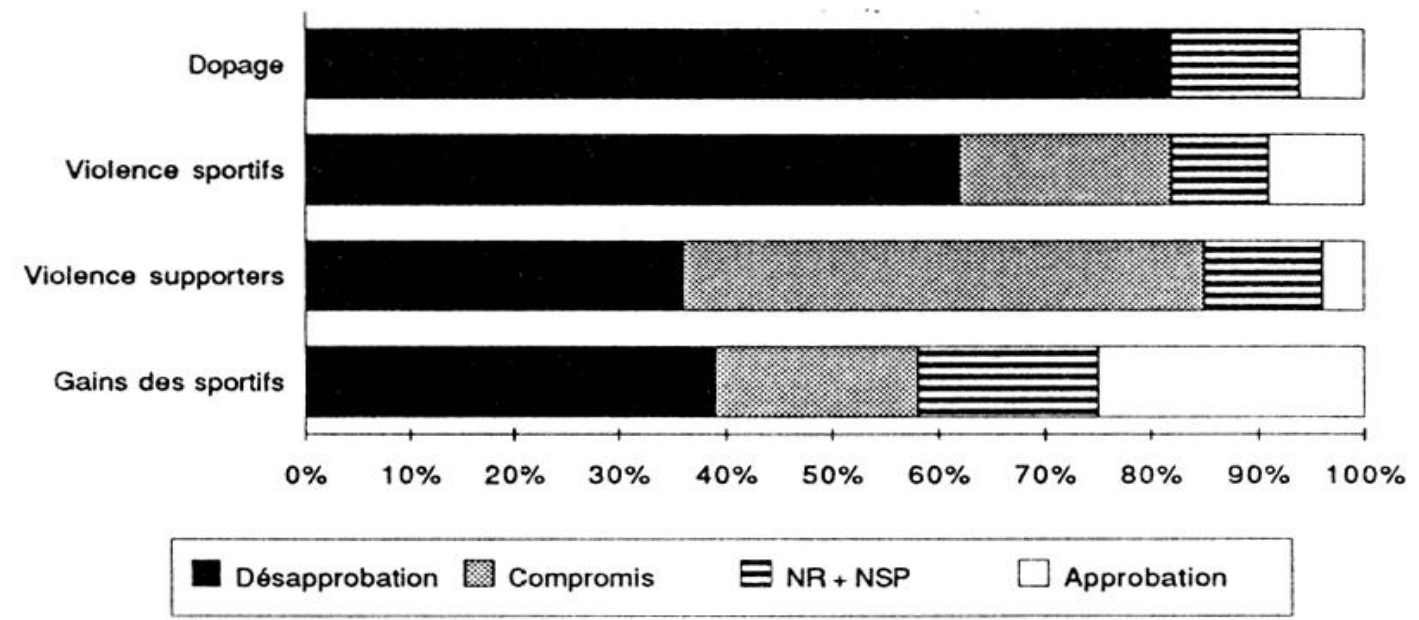

Fig. 1. - Attitude des jeunes face aux grands thèmes de l'éthique sportive

1. Pour chaque thème, les jeunes pouvaient ne pas se prononcer, approuver ou désapprouver le phénomène, ou encore adopter une attitude de compromis : par exemple, concernant la violence, la désapprouver mais penser qu'elle est inévitable. 
D'une manière générale, seule une minorité d'enfants approuve les pratiques condamnées par l'éthique sportive traditionnelle. La plus forte convergence se réalise autour du rejet, presque unanime, du dopage. La violence des sportifs est désapprouvée par la grande majorité des jeunes, mais $20 \%$ la pensent inévitable. En ce qui concerne la violence des supporters, les opinions de compromis prennent le pas sur la désapprobation : un peu plus de la moitié des jeunes qui ont exprimé une opinion réprouvent cette violence mais pensent qu'il est difficile de l'empêcher. Quant aux gains des sportifs, seulement un tiers des jeunes les condamnent. Il est vrai qu'aujourd'hui, le débat entre sport amateur et sport professionnel est dépassé, l'argent faisant désormais partie intégrante du système sportif.

L'engagement idéologique des jeunes varie selon leurs caractéristiques sociodémographiques. La désapprobation de ces divers phénomènes augmente nettement avec l'âge évalué par le niveau scolaire (cf. tableau 5) tandis que le nombre des non-réponses décroît. Pour porter un jugement sur une pratique, il faut évidemment savoir de quoi il est question. Avec l'âge, les sources d'informations s'accroissent, et plus généralement les connaissances et la capacité de s'informer. L'éthique sportive suppose une culture sportive ; cette dernière s'édifie progressivement. Avec l'âge, on constate une prise de position plus fréquente, mais on repère également une adhésion croissante à l'éthique sportive qui suggère une entrée progressive dans l'idéologie du sport.

Tableau 5. - Approbation selon le niveau scolaire (en \%)

\begin{tabular}{|l|c|c|c|}
\hline \multicolumn{1}{|c|}{ THEMES } & PRIMARE & COMEGE & LYCEE \\
\hline Gains des sportifs & 26,4 & 25 & 21,2 \\
\hline Violence supporters & 9 & 6,6 & 4 \\
\hline Violence sportifs & 10,7 & 5,9 & 4,3 \\
\hline Dopage & 7,4 & 5,6 & 2,5 \\
\hline
\end{tabular}

Face à l'éthique sportive, filles et garçons montrent des attitudes un peu différentes. Les garçons sont plus nombreux à exprimer une opinion; leur intérêt plus marqué pour l'information et le spectacle sportif est repérable dès l'âge scolaire. Si les filles sont moins bien informées sur le sport et si elles sont plus nombreuses à réserver leur jugement, elles apparaissent néanmoins comme les "gardiennes" des valeurs traditionnelles de l'éthique sportive (cf. tableau 6).

Tableau 6. - Approbation selon le sexe (en \%)

\begin{tabular}{|l|c|c|}
\hline \multicolumn{1}{|c|}{ THEMES } & FIUES & GARCONS \\
\hline Gains des sportifs & 21,3 & 28 \\
\hline Violence supporters & 3,4 & 5,2 \\
\hline Violence sportifs & 7,3 & 9,9 \\
\hline Dopage & 5,4 & 7,1 \\
\hline
\end{tabular}


L'adhésion aux grands principes de l'éthique sportive se différencie également selon les milieux sociaux. Manifestement, ce sont les groupes sociaux les plus aisés qui sont les plus réceptifs à la culture et à l'éthique sportives : viennent en tête pour la condamnation radicale du dopage $(76 \%)$, de la violence des sportifs $(68 \%)$ et de celle des supporters $(47 \%)$, les enfants des membres des professions intermédiaires, suivis par ceux des cadres et des professions intellectuelles supérieures. Ces derniers sont les plus nombreux $(40 \%)$ à condamner les gains des sportifs. Il faut souligner que ces groupes sociaux sont les mieux dotés en capital scolaire et culturel, c'est-à-dire qu'ils disposent sans doute du plus important "bagage " d'informations. Mais il faut rappeler également qu'ils sont les plus engagés dans la pratiquc sportive. Or, l'adhésion aux grands principes de l'éthique sportive croît globalement avec la durée hebdomadaire de la pratique et ce d'autant plus qu'elle est effectuée au sein d'un club. Il faut néanmoins nuancer ce constat : l'approbation du dopage s'avère plus forte chez ceux qui pratiquent du sport cinq heures et plus par semaine $(6,4 \%)$ que par ceux qui en font trois à quatre heures $(4,9 \%)$.

L'ensemble de ces résultats montre que la grande majorité des enfants que nous avons interrogés adhèrent largement à l'éthique sportive, et ce d'autant plus qu'ils sont eux-mêmes engagés dans la pratique. Les normes véhiculées par le monde sportif semblent donc bien intériorisées. Si l'engagement des jeunes dans l'éthique sportive ne fait aucun doute, ceux-ci font néanmoins montre d'un certain réalisme. Les gains des sportifs sont relativement peu réprouvés, de même qu'ils ne sont plus condamnés par le monde sportif. Concernant la violence des sportifs et des supporters, beaucoup la désapprouvent mais la pensent inévitable. En revanche, le consensus est très fort pour rejeter la pratique du dopage, fortement prohibée par le monde politique et sportif. L'opinion des jeunes apparaît donc comme un compromis entre la «morale coubertinienne » et la réalité sociale du sport véhiculée par les mass media : ils ont intégré l'éthique sportive mais elle ne domine pas leur jugement au point de les couper des réalités.

III - LES ENFANTS ET LES CHAMPIONS

Parce que la socialisation n'est pas seulement une transmission de valeurs, de normes et de règles mais passe aussi par un processus d'identification, les champions admirés par les enfants $(81 \%$ d'entre eux souhaiteraient en rencontrer) constituent un objet d'étude important. Les champions sportifs ne sont pas les seuls héros des enfants. Cependant, l'enquête a permis de montrer qu'ils arrivent au premier rang des popularités, précédant les chanteurs et les acteurs.

Le grand nombre de héros cités (près de 500 noms ont été avancés) renvoie à une diversification des références; le héros absolu n'existe pas. Dans 
cet Olympe cosmopolite se côtoient des personnages aux statuts différents : on y trouve aussi bien les grands héros portés par les médias que les vedettes locales (Metoudi, 1993). Papin, Platini, Prost, Arthaud sont les champions les plus cités (cf. fig. 2) ; sous l'angle de la notoriété, ils apparaissent donc comme les plus grands'.

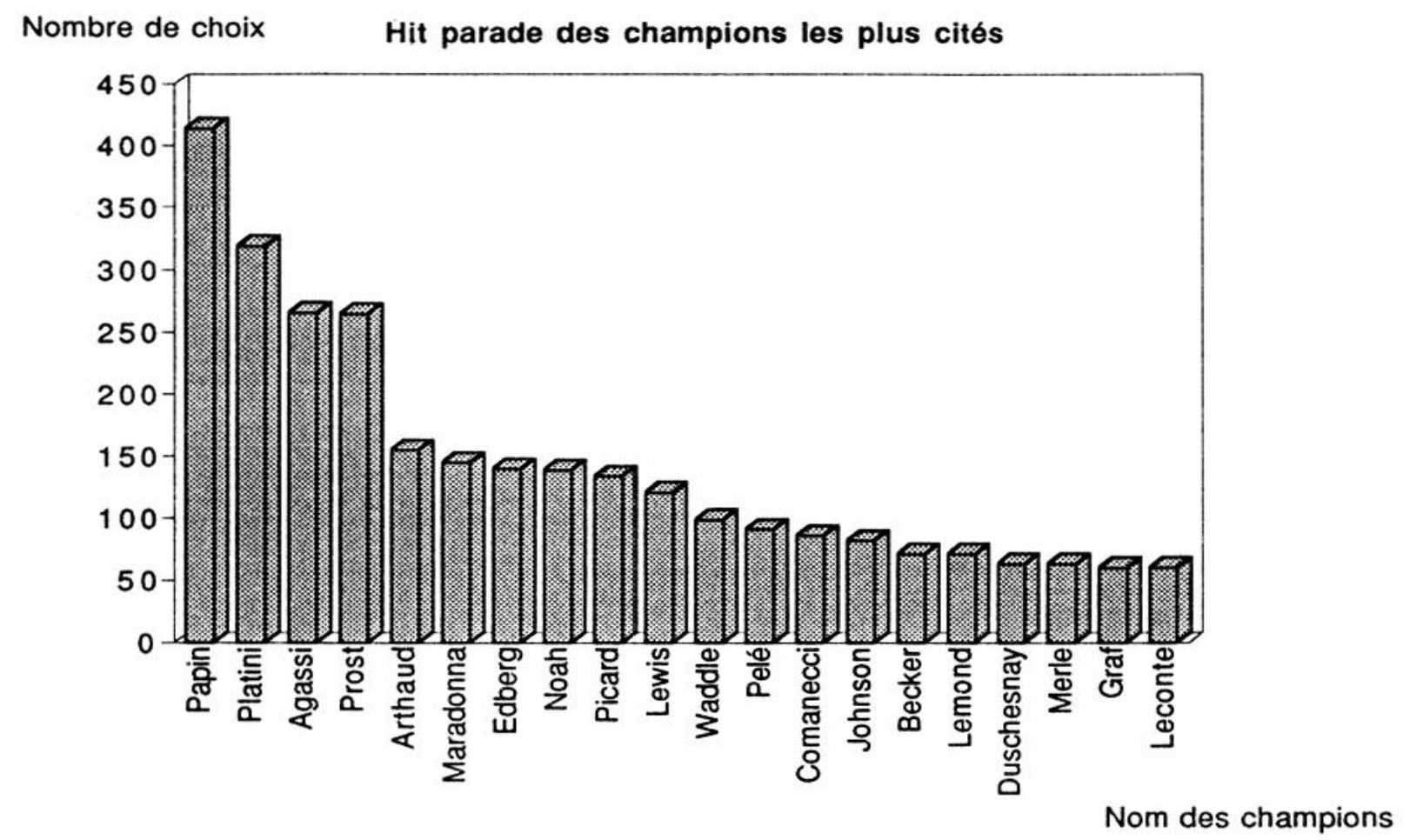

Fig. 2. - Hit-parade des champions les plus cités

Les fréquences de choix ne relèvent pas, à l'évidence, de l'influence de l'éducation physique et sportive scolaire. En comparant les temps d'enseignement consacrés à chaque discipline sportive (Groupe d'innovation pédagogique, 1991) aux champions cités par les enfants, on constate que leur culture sportive s'éloigne toujours davantage de celle de l'école. Le tennis arrive en tête des choix alors qu'il est absent, sauf exception, du temps d'enseignement (il en est de même pour la voile, le cyclisme ou la formule 1). La culture de l'élève se construit par des canaux extérieurs à l'école qui devrait au moins en mesurer l'importance. En revanche, l'influence de la télévision semble déterminante sur le palmarès des champions, ce dernier suivant assez fidèlement le temps d'antenne qui leur est consacré. Le tennis et le football, sports les plus

1. L'enquête a été réalisée fin 1990 ; à ce moment, l'Olympique de Marseille, engagé en Coupe d'Europe, était omniprésent dans les médias, de même que F. Arthaud, qui venait de remporter la " Route du Rhum ». 
présents à la télévision, rassemblent six champions parmi les dix premiers choisis par les enfants.

La télévision émiette la communauté en autant de cellules intimes composées d'enfants à la fois massifiés et séparés qui peuvent relier et faire cohabiter, dans leurs représentations, les "grands " héros de l'écran" et les " petits " héros du quartier. Les enfants jouent en permanence sur un univers culturel à deux dimensions, locale et planétaire : leurs héros proviennent aussi bien du bout de la rue que des quatre coins du monde.

Cet état de fait entraîne plusieurs conséquences. Tout d'abord, les héros gagnent en proximité. Les enfants les traitent avec familiarité, ce sont leurs " potes ». Quand Noah fait place à Yannick ou Leconte à Riton, l'inaccessible devient plus proche par l'usage du prénom ou du surnom. Ensuite, les héros ne se détachent plus de la communauté. Ils incarnent la promotion de l'homme ordinaire (Ehrenberg, 1991). Près de $50 \%$ des enfants estiment que « les champions sportifs sont des gens comme les autres "; seulement $8 \%$ les considèrent comme «des stars inaccessibles ». Du coup, les enfants passent parfois des coulisses à la scène et peuvent être choisis pour héros par leurs pairs. Quand ils retiennent un camarade "déclassé " ${ }^{2}$, la taille de l'exploit compte moins que la marque personnelle qu'il lui imprime. Enfin, les identifications ont acquis plus de multiplicité, plus d'errance, plus de fractionnement. Les lieux d'élaboration d'un sens collectif (consensuel ou transcendant) discuté ou incorporé sont rendus moins efficaces par le fonctionnement même de la communication.

La discipline sportive pratiquée par les enfants influence le choix des champions qu'ils aimeraient rencontrer. Ainsi, les jeunes tennismen citent davantage Edberg (11,5\%), Leconte $(10,2 \%)$ ou Agassi $(7,3 \%)$; c'est vers Papin $(25,3 \%)$, Platini $(17,7 \%)$ ou Maradonna $(8,3 \%)$ que vont les faveurs des jeunes footballeurs. Néanmoins, certains champions suscitent un intérêt qui vaut même pour les non-spécialistes. Ainsi, $7 \%$ des jeunes skieurs espèrent rencontrer Agassi ou Papin alors que $6 \%$ souhaitent voir C. Merle. Là encore on peut voir les effets d'une société médiatique où le charisme du champion, amplifié par les images, subjugue les enfants bien au-delà de leur pratique personnelle.

L'ensemble de ces résultats soulève des problèmes éducatifs majeurs si l'on s'en tient à une conception de la socialisation reposant sur l'intériorisation de normes extérieures.

D'abord, les enfants ont une culture sportive essentiellement bornée au contemporain : les héros choisis l'illustrent à l'évidence. Les grands champions des années passées ont gravi les marches des podiums sans y laisser d'empreinte. Or, l'éducation est en charge de travailler sur la mémoire collec-

1. Certains sports soulèvent le problème de la cohabitation entre les héros en chair et en os et ceux de dessins animés : c'est le cas du volley-ball où entrent en concurrence Alain Fabiani et « Jane et Serge » (Duret, 1993).

2. Pour les enfants, cette expression est un superlatif signifiant que l'individu échappe à tout système de classement. 
tive. Il s'agit de la part des enfants sans doute plus d'une ignorance que d'une amnésie. Cependant, rappelons que jamais comme aujourd'hui les héros n'ont été aussi éphémères, aussi vite chassés qu'arrivés, dévorés par une société boulimique d'images.

Ensuite, on est bien obligé de reconnaître que la transgression d'interdits sociaux n'affecte guère, aux yeux des enfants, les images de B. Johnson ou de D. Maradonna. Tout deux figurent parmi les quinze premiers champions que les enfants aimeraient rencontrer.

Enfin, les enfants expriment un modèle héroïque où l'argent fait partie des toutes premières attentes légitimes associées à l'excellence sportive. L'estime de soi qui se développe à travers le jcu des identifications s'appuie sur des bénéfices escomptés d'ordre relationnel mais d'une manière moins déterminante que sur la reconnaissance offerte par l'argent. Pour ces jeunes, devenir riche constitue un argument majeur de l'attrait à devenir champion avant même de songer à avoir beaucoup d'amis ( $86 \%$ invoquent le premier argument, $66 \%$ se réfèrent au second).

\section{CONCLUSION}

Dire que le sport socialise les jeunes nécessite de s'entendre sur chacun des termes : sport, socialisation et jeunes.

La première limite de ce travail concerne le sport qui ne saurait se réduire au sport institutionnalisé. Celui-ci constitue aujourd'hui un objet extrêmement composite. Qui a côtoyé les finales du championnat de France et le foot des parkings, le faste des grands gymnases et le dénuement des banlieues, la compétition exacerbée et l'amusement insouciant, aura sans doute quelque peine à accepter que le même mot de sport puisse caractériser des situations de socialisation aussi dissemblables. La transmission des valeurs dépend donc grandement des modalités de pratique choisies.

La seconde limite est d'ordre méthodologique. Le point fort de l'enquête est qu'elle touche un grand nombre de jeunes; en contrepartie, elle ne nous permet d'évaluer que le versant de la socialisation concernant l'intériorisation de normes extérieures ${ }^{1}$ et peu celui où les valeurs sont étudiées dans l'in-

1. Il faut rappeler que cette approche durkheimienne de la socialisation a fait l'objet de critiques venant de la psychologie, de la sociologie et de la philosophie. En psychologie, Piaget (1932) lui reprocha d'ignorer les effets du développement de l'enfant et de ne pas prendre en compte les stades qui semblaient caractériser la formation du jugement moral. En sociologie, les ethnométhodologues (Garfinkel, 1967) prônent que les normes morales ne valent que forgées dans l'interaction. Les valeurs n'existent plus a priori mais se construisent dans l'échange. Enfin, en philosophie, la principale objection portée au paradigme durkheimien consiste à dire que toute véritable morale est personnelle et intime, et qu'elle ne peut donc pas faire l'objet d'une imposition venue de l'extérieur sans aller à l'encontre du but escompté (Ladrièdre, Gruson, 1992). 
teraction sociale. Dans cette seconde perspective, des recherches menées au laboratoire de sociologie de l'INSEP (Duret et Augustini, 1993), auprès de volleyeurs de 10 à 14 ans et de footballeurs de 10 à 11 ans, ont montré que pour les jeunes, la justice sportive pouvait être le fruit d'une combinaison de principes (don, droit, effort...) en délibéré au gré des situations (formation de l'équipe, emprunt et gestion de matériel...).

Enfin, la dernière limite touche à la définition de la jeunesse qui n'est pas, d'un point de vue sociologique, une catégorie unifiée. Que de différences entre la jeunesse traditionnelle qui partage son temps entre la famille, l'école et le club et la jeunesse en difficulté soumise à la " galère "! Ainsi, si la pratique sportive est amplement répandue chez les jeunes, elle ne garantit aucunement l'adhésion aux structures sportives traditionnelles. Les adeptes du streetball restent, par exemple, très majoritairement réfractaires au club ou à l'association sportive scolaire. L'enquête ayant été réalisée à l'intérieur des établissements scolaires, et dans le cadre d'un concours, les enfants qui y ont répondu appartiennent à la jeunesse traditionnelle, bien insérée scolairement et socialement.

Par-delà ces limites, les résultats de cette enquête mettent en évidence à la fois des différences et des consensus témoignant que des modèles sociaux relevant de l'environnement proche et quotidien influencent les pratiques et opinions des enfants. A de nombreux égards, on voit apparaître chez eux le produit d'une " pédagogie implicite " (De Singly, 1987). C'est l'intensité et le degré d'institutionnalisation (en particulier chez les plus jeunes) qui différencient la pratique des enfants de celle des adultes, plus que le choix des sports en lui-même qui, n'échappant pas au marquage socioculturel, suggère nettement une reproduction sociale des goûts et des pratiques.

Du point de vue de l'éthique, la frontière entre ce qui est accepté ou dénoncé suit la ligne de démarcation entre ce qui est permis et prohibé. L'originalité de cette position est qu'elle s'accompagne d'une vision réaliste où les jeunes peuvent réprouver le dopage ou la violence tout en les considérant comme inévitables.

Quant aux champions, s'ils constituent toujours des figures propices à l'identification, ils ont gagné en proximité. Celle-ci conduit à les désacraliser, à les dépouiller de leur auréole mythique. Les champions ne valent plus comme des repères au-dessus de la mêlée.

Le sport ne peut plus être considéré comme une vaste machine à socialiser. L'une de ses principales fonctions idéologiques a été de fournir des modèles de citoyenneté, de santé, de justice... le Heysel, Ben Johnson, ou encore l'affaire oM-Valenciennes illustrent aujourd'hui la faiblesse et l'impuissance de telles promesses. Pour prétendre à une réelle efficacité, la pratique sportive des enfants doit passer par une diversité d'instances et de procédures qui, par-delà les discours, leur font vivre au quotidien le processus de socialisation. 


\section{BIBLIOGRAPHIE}

Bozon M., Les loisirs forment la jeunesse, in Données sociales 1990, Paris, INSEE, 1990.

Davisse A., Louveau C., Sport, école, sociéłé : la part des femmes, Joinville-le-Pont, Actio, 1991.

De Singly F., Fortune et infortune de la femme mariée, Paris, PUF, coll. "Economie en

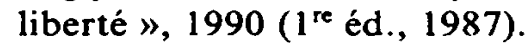

Duret P., L'héroisme sportif, Paris, PUF, 1993.

Duret P., Augustini M., Sports de rue et insertion sociale, Paris, INSEP, 1993.

Duret P., Irlinger P., Louveau C., Vulbeau A., Le sport, moi et les autres : pratiques et représentations du sport chez les jeunes, Paris, Syros/Alternatives, 1993.

Ehrenberg A., Le culte de la performance, Paris, Calmann-Lévy, 1991.

Forquin J.-C., L'enfant, l'école et la question de l'éducation morale, Revue française de pédagogie, $\mathrm{n}^{\circ} 102,1993$, p. 69-106.

Galland O., Sociologie de la jeunesse, Paris, Armand Colin, 1991.

Garfinkel H., Studies in Ethnomethodology, New York, Polity Press, 1967.

GIP ( Groupe d'innovation pédagogique) de l'académie de Créteil, Quels programmes pour quels élèves?, document destiné aux contributions rendues publiques par l'Inspection générale de l'EPS, avril 1991 (document ronéoté).

Irlinger P., Louveau C., Metoudi M., Les pratiques sportives des Français; enquête sur les usages sportifs du temps libéré, Paris, INSEP, 1987, 2 t.

Ladrièdre P., Gruson C., Ethique et gouvernabilité, Paris, PUF, 1992.

Lipovetsky G., L'ère du vide, essai sur l'indivisualisme contemporain, Paris, Gallimard, 1983.

Metoudi M., La place des femmes dans l'héroïsme sportif, Esprit, septembre 1993, p. 44-68.

Piaget J., Le jugement moral chez l'enfant, Paris, PUF, 1932.

Vigarello G., Le corps redressé, Paris, Delarge, 1977. 